\title{
Mal uso del suelo en Colombia ocasiona problemas socioeconómicos*
}

\section{Wrong use of the soil in Colombia cause of socioeconomic problems}

\author{
Serrato Vásquez Maritza ${ }^{1}$ \\ ${ }^{1}$ Licenciada en Producción Agropecuaria, Universidad de los Llanos. \\ *Ensayo realizado en cursos posgrado "Especialización Producción Agrícola \\ Tropical Sostenible" de UNILLANOS, presentado como requisito en la para optar \\ al título de Licenciada en Producción Agropecuaria
}

\section{maritza.serrato@unillanos.edu.co}

Recibido 12 de Junio 2013, Aceptado 15 de Julio 2013

\section{RESUMEN}

Los recursos naturales son los elementos y fuerzas de la naturaleza que el hombre puede utilizar y aprovechar y además representan fuentes de riqueza para la explotación económica, pero el mejor aprovechamiento de un recurso depende del conocimiento que tenga el hombre sobre las leyes que rigen la conservación de los recursos naturales. El manejo sustentable es una preocupación mundial y la producción de alimentos es una necesidad básica fundamental para garantizar la seguridad y soberanía alimentaria de los habitantes de un territorio, por lo cual es de gran importancia el uso racional del suelo y el agua. Actualmente se observa que el principal objetivo del ser humano cuando posee tierras, en la explotación excesiva, es el de obtener ganancias económicas rápidas que le garanticen una calidad de vida superior a la que posee, relegando el uso adecuado del suelo; esto conlleva a que en Colombia el total de tierras sobreutilizadas en grado severo y moderado sea más del 60\%, especialmente en actividades agropecuarias, en zonas recomendadas para uso forestal de protección y producción, con el fin de lograr conservación y recuperación integral de los recursos hídricos. Los productores en Colombia están acostumbrados a estar rodeados de una amplia biodiversidad, gozando de un ambiente optimo donde se puede obtener cualquier producto agropecuario, además consideran que siempre van disfrutar de estas ventajas, lo que conlleva al manejo irracional, dando como resultado paisajes de desertificación, suelos erosionados, depredación de flora y fauna silvestre, 
contaminación en cuencas hídricas, ocasionando pobreza socio-económica debido a un desequilibrio incontrolable en el ambiente, con el temor de que estos procesos sean de recuperación difícil o irreversible. Este ensayo concluye con las soluciones que las entidades ambientales han propuesto y que como ciudadanos profesionales del sector agropecuario se puede brindar apoyo para contribuir en el mejor manejo y conservación del recurso más importante, pero igualmente el más olvidado "EL SUELO".

Palabras clave: Suelo, ambiente, producción agropecuaria.

\begin{abstract}
Natural resources are the elements and forces of nature that man can use and benefit and also represent sources of wealth for economic exploitation. But the best use of a resource depends on the knowledge that man has on the laws governing the conservation of natural resources. Sustainable management is a global concern and food production is a fundamental basic need to ensure food security and sovereignty of the people of a territory, by which great importance is the rational use of land and water. Currently it appears that the main aim of human when landless is exploitation, is the rapid economic gains that guarantee a quality of life than man has, relegating the appropriate land use, this means that in Colombia on the total land used in severe and moderate degree is over $60 \%$, especially in agricultural activities in areas recommended for forest protection and production in order to achieve comprehensive conservation and restoration of water resources. Farmers in Colombia are used to being surrounded by a large biodiversity, enjoying an optimal environment where you can get any agricultural product, and will always consider these benefits, leading to irrational management, resulting in landscape desertification, eroding soils, depletion of wildlife, watershed pollution, causing socioeconomic poverty uncontrollable due to an imbalance in the environment, with the fear that these processes are difficult or irreversible recovery. The paper concludes with the solutions that have been proposed environmental entities and as citizens agricultural professionals can provide
\end{abstract}


support to contribute to the better management and conservation of the most important resource, but also the most forgotten "SOIL".

Keywords: Soil, environment, agricultural production.

\section{INTRODUCCIÓN}

Actualmente se observa que el principal objetivo del ser humano cuando posee tierras, en la explotación excesiva, es el de obtener ganancias económicas rápidas que le garanticen una calidad de vida superior a la que posee, relegando el uso adecuado del suelo (Malagón, 2002). A través del mundo en desarrollo, el cambio climático, la reemergencia de la volatilidad de los precios en los alimentos, la degradación constante de los recursos naturales y otras amenazas han agravado el hambre y la pobreza; mientras amenazan también los ecosistemas de donde proceden los medios de vida de la población rural (CIAT, 2009).

En países latinoamericanos cerca del $50 \%$ del suelo es usado de manera inapropiada, esto puede traer consecuencias graves dado que es una de las principales causas de su degradación. A menudo se encuentran casos de conflicto como el potencial agrícola empleado en bosques secundarios o viceversa; parte de este problema reside en la carencia de información precisa acerca de las características físicas, químicas y biológicas de los suelos de manera que el campesino tenga conocimiento para dar un manejo adecuado de este recurso de acuerdo a sus potencialidades (Rodríguez, 2005).

No es solo el hecho de realizar un mal manejo de los recursos que nos brinda la naturaleza, sino que también se está afectando la economía de un país, convirtiéndose de esta manera en un problema ambiental, social y económico; por ende, al poseer tierras infértiles no hay trabajo ni alimento, incrementando así la pobreza en nuestros países.

\section{ANÁLISIS DE LA SITUACIÓN EN COLOMBIA}

Colombia ha sido un país con suficientes recursos naturales, pero ya se comienza a notar en parte sustancial del territorio la angustia de la escasez, el impacto del 
deterioro del suelo, la contaminación del ambiente, la desaparición definitiva de especies forestales, la presencia de construcciones en zonas por excelencia de alta producción agrícola, el hacinamiento de gente en las ciudades, la presencia de plagas y enfermedades de especifica virulencia en cultivos y ganado, el aumento de inundaciones, derrumbes, sequias intensas, atribuible en gran medida al mal uso de la tierra y explotación irracional de la vida silvestre (Lombo, 1998). La problemática anteriormente mencionada se debe en gran medida a que existe un inadecuado ordenamiento territorial, utilizando áreas de alto valor agrícola para construcción de urbanizaciones cuando de hecho se sabe que es prioritaria la producción de alimentos, que son escasos mientras la población se incrementa significativamente cada día, conduciendo a la sociedad, a una pobreza extrema donde escasamente se logra conseguir para uno o dos alimentos diarios. Esta situación es crítica debido a que se ha acrecentado la degradación de los recursos naturales y la contaminación ambiental, problemas que se pueden observar con mayor incidencia en los trópicos. Por esta razón las autoridades ambientales, han tomado precauciones como la clasificación de los usos restringidos del suelo delimitando reservas forestales, encargadas de brindar un desarrollo sostenible en las partes que se localizan los abastecimientos; principalmente de agua que son vitales para la supervivencia del ser humano.

El adecuado uso del suelo, se hace entonces una herramienta de vital importancia para el establecimiento de soluciones que contribuyan a la retroalimentación entre medio ambiente y campesino, ya que al mantener un equilibrio adecuado entre los dos se puede crear una simbiosis que garantice el mantenimiento y conservación de los recursos naturales, así mismo es necesario lograr que el campesino no pierda su sostenibilidad económica que le es brindada por su finca. El uso del suelo, es uno de los principios determinantes en lo relacionado con el desarrollo de políticas ambientales en los países con economías primarias (Rodríguez, 2005).

En Colombia, los campesinos han sido relegados a las peores tierras y han tenido que adaptar sus posibilidades productivas a estas condiciones. Sin embargo, han 
mantenido una amplia participación en el control de áreas dedicadas a cultivos, en el mercado agroalimentario y en la generación de valor (Corrales, 2001). Comenzando de esta manera una rivalidad entre el campesino y las instituciones protectoras del medio ambiente, por mal manejo de los recursos en estas zonas que cuentan con pendientes aproximadas del $25 \%$, por tal motivo las instituciones protectoras del medio ambiente sellan las explotaciones agropecuarias, basándose en argumentos como deforestación, y mal uso de desechos, logrando que los campesinos de estas zona los vean como agentes opresores y dictadores que intentan acabar con el abastecimiento económico con el que han subsistido durante varios años, en este punto se requiere buscar alternativas educativas que contribuyan en la creación de conciencia ambiental y el uso adecuado del suelo que es la falla más grande que posee la población Colombiana (CORPAMBU, 2008).

Las entidades ambientales sostienen que los campesinos de estos lugares pueden acceder a la utilización de sus tierras con el compromiso de utilizar nuevas tecnologías que permitan controlar y disminuir la contaminación, erosión y sobreexplotación de la tierra, los propietarios no han sido abiertos a la adquisición de nuevas tecnologías debido a que éstas incrementarían sus gastos, y muchos de ellos no cuentan con los recursos financieros necesarios para adaptar sus fincas a estas exigencias. Por lo tanto, estas entidades encargadas del medio ambiente deberían promover y apoyar el buen uso de los sistemas tradicionales considerados conservacionistas caracterizados por ser Integrales, igual que el conocimiento íntimamente relacionado, empleando la base cultural que lo sustenta para lograr conservar los recursos naturales en zonas de asentamientos indígenas y campesinos. Entendiendo correctamente las características integrales de este procedimiento, el campesino podrá hacer un manejo adecuado con el medio ambiente, como en los tiempos antiguos donde tanto el hombre como la naturaleza generaban un intercambio de bienes y servicios proporcionando un contexto sostenible y productivo (Corrales, 2001). 
La identificación de la producción campesina como eminente conservadora de biodiversidad, es un punto que está a discusión, especialmente para aquellos sectores cada vez más vinculados al mercado y a la producción con sistemas que utilizan una alta proporción de insumos externos como semillas, agroquímicos y fuentes de energía no renovables, concerniente a que al campesino de hoy, no le interesa cuanto le va a durar su producción si no por el contrario buscan encontrar una rentabilidad rápida y eficiente. Aun así, se puede considerar que existe algún manejo de biodiversidad, entre otros: utilización de cultivos diversificados, la integración de actividades agrícolas y pecuarias, en sistemas de rotación de cultivos y asocio entre cultivos (Marcos, 1996).

\section{CUESTIONAMIENTO DEL USO DE SUELO EN COLOMBIA}

El suelo hace parte de los recursos naturales de un país, con igual importancia que el agua y los bosques, no obstante, frecuentemente recibe menos atención, por eso, dependiendo de estudios realizados por instituciones encargadas como el Agustín Codazzi, se pueden observar la vocación para la que está dispuesta cada zona, dependiendo su ubicación y climatología (Tabla 1).

Tabla 1. Distribución del uso del suelo en Colombia

\begin{tabular}{|c|c|}
\hline Actual & Recomendado \\
\hline \multirow{3}{*}{ Agrícola } & Cultivos transitorios intensivos \\
\hline & Cultivos transitorios semi-intensivos \\
\hline & $\begin{array}{l}\text { Cultivos semipermanentes y permanentes intensivos. } \\
\text { Cultivos semipermanentes y permanentes semi-intensivos } \\
\text { Silvoagrícola }\end{array}$ \\
\hline \multirow[t]{2}{*}{ Agroforestal } & Agrosilvopastoril \\
\hline & Silvopastoril \\
\hline Ganadería & $\begin{array}{l}\text { Pastoreo semi-intensivos y intensivo } \\
\text { Pastoreo extensivo }\end{array}$ \\
\hline \multirow{2}{*}{ Forestal } & Producción \\
\hline & $\begin{array}{l}\text { Protección - producción } \\
\text { Forestal de protección }\end{array}$ \\
\hline \multirow[t]{2}{*}{ Conservación } & Recursos hídricos e hidrobiológicos \\
\hline & Recuperación \\
\hline
\end{tabular}

Fuente: IGAC, 2012. 
El manejo que actualmente se está brindando a los suelos colombianos es muy diferente a la vocación correspondiente de cada uno, se observa degradación creciente de los recursos naturales como un aspecto que hoy en día ocupa la agenda no solo de los organismos del estado, encargados de velar por su conservación, sino que ha trascendido al conjunto de la sociedad, convirtiéndose en motivo de preocupación pública. Se han hecho investigaciones de esta problemática de las tierras en Colombia en lo que concierne a la presión y características de uso del territorio, actualizando conceptos de cartográfica en cobertura y uso actual de las tierras, estableciendo la vocación actual (Figura 1) como los conflictos sobre el manejo y uso de las tierras (IGAC, 2012).

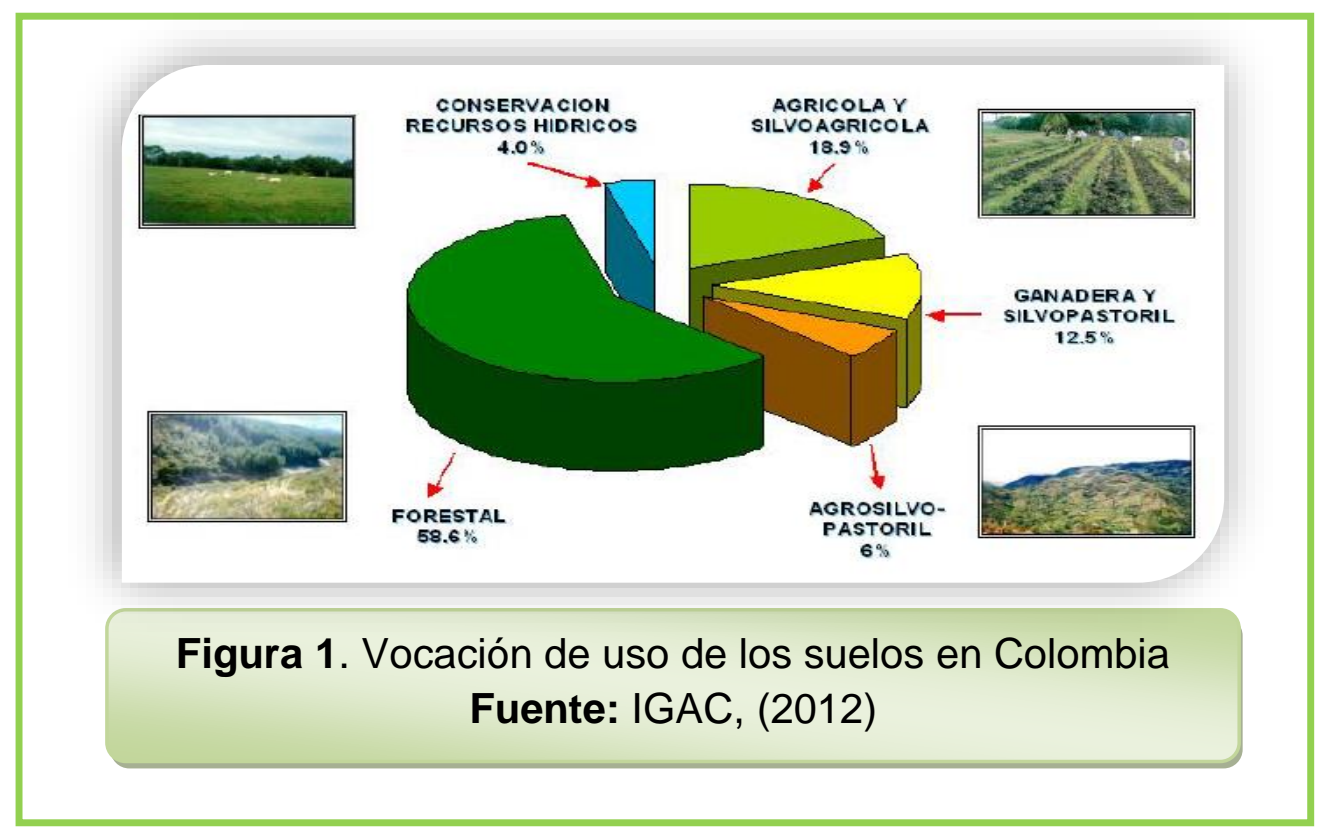

El potencial actual agropecuario del país se aproxima al $37 \%$ del territorio y comprende tanto los sistemas tradicionales como los integrados en el bosque, es decir silvopastoriles, silvoagrícolas y agrosilvopastoriles. Ello implica, el desarrollo de paquetes tecnológicos, de investigación y de preparación profesional con la finalidad de adelantar adecuadamente su implantación (Figura 1). Lo anterior permite evidenciar el potencial del país en este aspecto siempre y cuando se solucionen los problemas nacionales; un hecho a resaltar es el daño productivo y físico al suelo debido a sobre o sub-explotación, causando pérdidas razonables a nivel productivo, económico y ambiental; caso contrario ocurre al momento de 
realizar un adecuado manejo al suelo, aseguraría la demanda de alimentos del país, además, sí se utilizan ventajas comparativas asociadas a su localización tropical y ecuatorial, generaría ganancias importantes vinculadas a la exportación de productos competitivos internacionalmente. Se evidencia que los suelos colombianos no están siendo utilizados según su vocación, sino de acuerdo a los requerimientos del productor; sin llegar a divisar que los recursos no son para suplir exigencias particulares, por lo tanto, es importante establecer un sistema de conservación y sostenibilidad.

Los mismos estudios realizados por el IGAC, (2012) indican que los terrenos sembrados con pastizales ocupan una extensión muy amplia, las dedicadas a la agricultura muy baja y los bosques han disminuido considerablemente lo que está afectando la diversidad biológica y por ende la productividad agropecuaria y medio ambiente, lo que repercute en aspectos socioeconómicos, en la calidad de los recursos y en el fomento de la erosión de tierras (Figura 2). Demostrando así, que en Colombia subsisten prácticas agropecuarias que deterioran el medio ambiente, como la administración de agroquímicos, la mecanización, la tala y quema de bosques como forma de 'adecuar' áreas de vocación forestal para la agricultura. Este tipo de prácticas ocasionan contaminación de aguas superficiales, erosión, compactación del suelo y por supuesto pérdida de diversidad biológica (Fandiño y Ferreira, 1998).

Por esta razón es que el uso de la tierra en el campo debe estar regida por la capacidad productiva y la aptitud agrícola de los suelos, por directrices concretas que separen la agricultura industrializada, de los minifundistas, entre los cuales existe una gran diferencia en el manejo de los recursos, mientras los primeros sobresaturan el suelo con la implementación de agroquímicos, los otros utilizan la tierra para el sostenimiento de su familia, aunque sin un adecuado manejo ambiental los dos sectores causan daños irremediables en el medio, ya sea en grandes o pequeñas escalas.

Las consecuencias de la sobreutilización de las tierras se reflejan principalmente en la degradación de los recursos naturales, cuya expresión más evidente es la 
erosión hídrica. En Colombia, el 35\% del total de las tierras se encuentran afectadas por erosión, con más de 4’300.000 hectáreas erosionadas severamente, y 12.916.000 hectáreas, en grado moderado (IGAC, 2012).

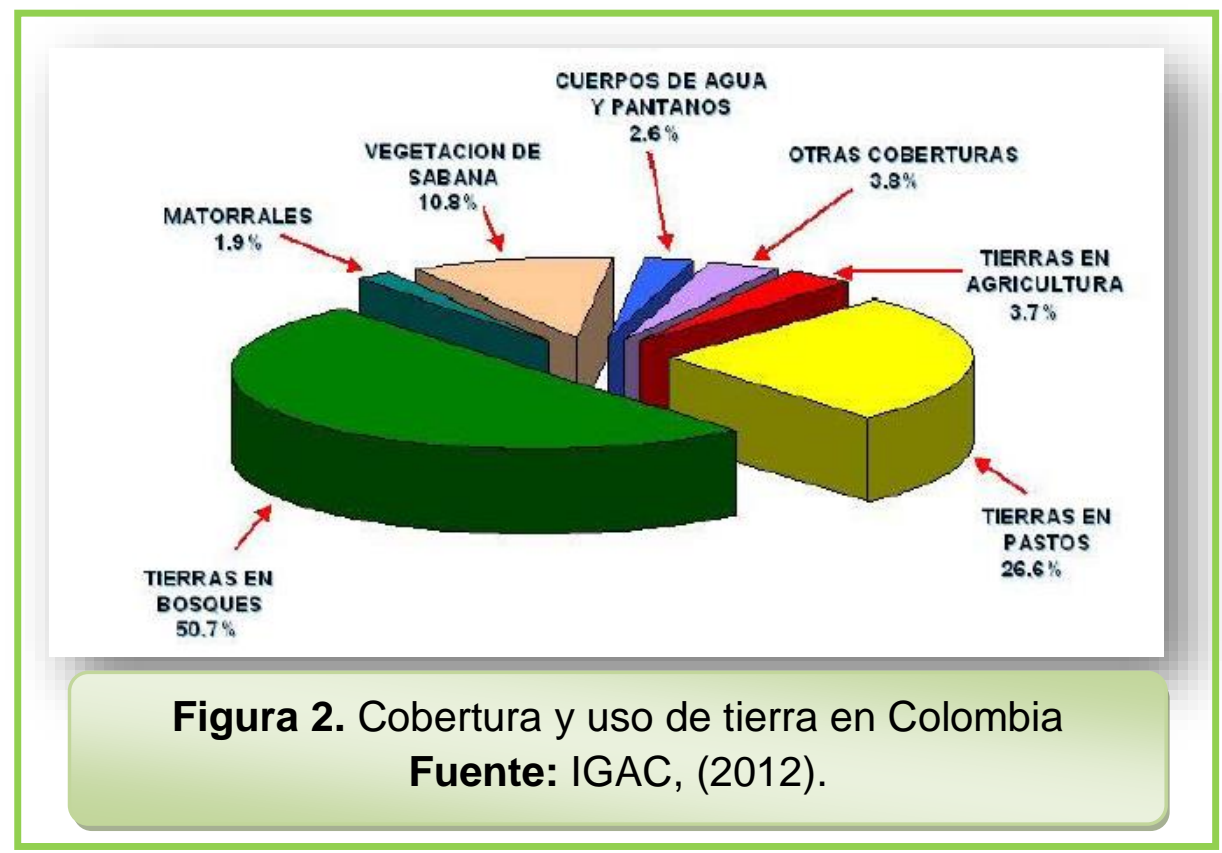

\section{PROBLEMÁTICA REGIONAL, VILLAVICENCIO (META)}

Como se ha especificado Colombia dispone de suficientes suelos y tierras que mediante la planificación y ordenamiento territorial pueden consolidar su seguridad alimentaria y producir recursos para la nación, dependiendo de sus ventajas comparativas y competitivas, relacionadas con los productos favorecidos por su localización en la zona intertropical y ecuatorial (Malagón, 2002).

La Diversidad de sus zonas son las que se deben tener en cuenta en el momento de realizar el ordenamiento territorial, asumiendo cuáles suelos son aptos para las diferentes explotaciones y así mismo aprovechar las características físicas que son proporcionadas por el ambiente para el fortalecimiento del sector productivo.

En la Resolución № 2350 de 2009 dada por el Ministerio de Medio Ambiente; identifica parámetros que permiten determinar las áreas de Villavicencio como Quebrada Honda, y Caños Parrado y Buque como "Zonas Forestales Protectoras", que por su nivel de precipitación y porcentaje de pendiente, la degradación de los 
suelos es elevada, por lo tanto deben mantenerse ambientalmente, para que cumplan con su función de protección de las corrientes hídricas. En esta legislación establece la importancia de generar corredores biológicos a 50 metros del lugar de donde hay una corriente fluvial, el otro terreno puede ser apropiado por los productores que posean una conciencia estructurada y centrada en la ecoeficiencia para dar un uso adecuado a su finca incluyendo los recursos naturales. El empleo de instrumentos de labranza amigables y de energía solar, eólica para que se disminuya paulatinamente el deterioro del suelo, la contaminación del agua y el empobrecimiento de la vida silvestre.

Las zonas estipuladas como reserva, en caso particular la reserva de Buenavista tienen la vocación del suelo como forestal protector, en el cual el decreto 1383 de 1940, determina que esta zona es el conjunto de terrenos que por su topografía o ubicación en las cabeceras de las cuencas hidrográficas, márgenes, depósitos y cursos de agua conviene que permanezcan revestidos de masas arbóreas por la acción que estas ejercen sobre el régimen fluvial, conservación de las aguas y suelos, salubridad de los centros urbanos, estableciendo restricciones para el aprovechamiento forestal de estas áreas y la adjudicación de terrenos baldíos (Resolución № 2350, 2009); de igual manera es de considerar problemas existentes en la ejecución de procedimientos agropecuarios que influyen en la falla ambiental, de esta forma no se justificaría desertar de un momento a otro gran cantidad de personas que por mucho tiempo han vivido de este terreno, sin recursos con que desenvolverse.

Pero lo que se observa en la actualidad es una gran desigualdad y equivocación en la ocupación de la tierra en los sectores rurales, lo q genera que las reformas agrarias que se han instaurado desde años atrás por muchos gobiernos se quedan solo en leyes o decretos, y tanto los pequeños como los medianos productores son trasladados hacia las laderas siendo de esta manera obligados a cultivar o sostener sus animales en pendientes fuertes como es en el caso de la Reserva Forestal de Buenavista, en la que se pueden encontrar agricultores con sus pequeñas parcelas en zonas de pendientes que no son aptas para esta clase 
de manejo, suelos que al ser sembrados se les quita la cubierta vegetal y árboles de gran tamaño provocando deslizamientos que lentamente van reduciendo el terreno apto para manejo de las fincas.

Villavicencio ubicado en el Piedemonte llanero no se excluye de este tema; está rodeado con un gran potencial forestal e hídrico encargado de suministrar agua e a sus habitantes, igualmente contribuye a la sostenibilidad de un suelo joven que está afectado por las diferentes fallas geológicas; áreas líticas y siempre deben protegerse con cubierta vegetal, ya que son paisajes productores de agua, que albergan una gran riqueza de fauna y flora silvestre. Este paisaje que bordea el municipio consta de: Quebrada honda, caños Parrado y Buque, y la "Reserva de Buenavista", constituida mediante la resolución número 59 de Abril de 1945 por el Ministerio de Economía Nacional, debido a la necesidad de conservar las cuencas hidrográficas que allí se originan garantizando de esta manera a futuro el suministro de agua para los habitantes del municipio, (Resolución № 2350, 2009).

Las fuentes superficiales de agua se han venido deteriorando año tras año, como consecuencia de la tala y quema indiscriminada en las zonas de hacimiento, igualmente por el mal manejo y administración de las cuencas aferentes, lo que ha conllevado a un desabastecimiento de agua para los acueductos, mayores costos para el suministro del líquido, inestabilidad de cauces y deterioro de los ecosistemas estratégicos acuáticos y terrestres, entre otros impactos; esta ley de conservación puede ser muy bien vista desde el sector ecológico, pero caso contrario sucede con los productores de estas tierras ya que se les prohíbe la producción agrícola y pecuaria.

Durante 60 años, muchas personas adquirieron tierras y crearon su minifundio como alternativa para mantener a sus familias, en el momento en que se reglamenta el uso de tierras, muchos campesinos se ven afectados porque desde tiempo atrás han explotado la tierra de manera artesanal utilizando sus ganancias como base esencial para su manutención y de esta forma proveer educación, alimentación y comodidades a sus hijos, además el municipio de Villavicencio al igual que la mayoría de municipios de Colombia sostiene un conflicto social y 
político, incrementando los frentes de colonización en áreas de alta biodiversidad, en particular en parques nacionales naturales (Cuellar, 2009).

El mal uso de las herramientas tecnológicas que se le han ofrecido actualmente al productor para su mejor desempeño, asociados con la intensa precipitación típica de la región, intensifica los daños de la estructura física del suelo que conducen al sellamiento de la capa superficial y la consecuente reducción drástica de las tasas de infiltración, flujo de aire y agua. Afectando la disponibilidad de nutrientes que generalmente son escasos en estos suelos.

Aunque Colombia cuente con grandes beneficios ambientales por ser parte de los países tropicales, el problema principal con las tierras es el desconocimiento de los factores formadores de los suelos, así como y el manejo inadecuado de los recursos naturales. En la solución del conflicto ambiental se ha descuidado la parte social. El énfasis se dirige hacia el desarrollo de los aspectos tecnológicos (Newhouse, 1990). Sin embargo, actualmente se reconoce que la tecnología por sí sola no puede solucionar los problemas ambientales, ya que parte de la crisis ambiental tiene sus orígenes en las percepciones y actitudes humanas hacia el medio ambiente (Barraza, 2001). La educación ambiental es una herramienta importante para la conservación de los recursos naturales, porque promueve y refuerza la participación dinámica y reflexiva hacia el mejoramiento del ambiente. Por esto se debe tener en cuenta la parte educativa. Se necesita formar recursos humanos orientados hacia el manejo de la restauración ecológica de los suelos, pero fundamentalmente con esquemas de pensamiento y formas de acción diferente, donde los campesinos y profesionales agropecuarios vean la tierra no solo como un medio de extracción, si no que generen una especie de simbiosis entre la productividad y la conservación; ya que "preservar los ecosistemas no es un mensaje que garantice la continua existencia como especie, se necesita pasar a vivir en cooperación con nuestro ecosistema, reintegrarnos a el de modo que el prefijo "eco" sea aplicado con relación a la especie Homo sapiens." (Marcos, 1996). 
El concepto de eco-eficiencia ha formado parte del discurso internacional sobre agricultura sostenible durante casi dos décadas, sin embargo, en los últimos años su importancia ha crecido, en la medida en las crisis han convergido para plantear retos importantes para la agricultura tropical, luego de pasar por una década donde primó la revolución verde que indujo a los campesinos a la utilización de químicos que incrementaron la erosión y perdida del suelo, ya que forzaban a suelos no aptos para la producción para el mantenimiento de determinadas especies agrícolas o pecuarias (CIAT, 2009).

\section{CONCLUSIONES}

De acuerdo con los estudios realizadas por el CIAT en el 2009, y como se había discutido anteriormente se pretende crear un campesino "eco- eficiente" que garantice el adecuado manejo de su finca, sobre todo si se encuentra en zonas de alto compromiso ambiental, y al mismo tiempo debe contribuir con las entidades del estado al sostenimiento productivo y económico de la población ya sea a nivel municipal o nacional, buscando obtener una agricultura que garantice bienestar integro, de la siguiente manera:

- Agricultura más productiva, proporcionando alimentos nutritivos de bajo costo para consumidores de escasos recursos, donde todas las familias obtengan un acceso constante a los recursos alimenticios, disminuyendo las tasas de desnutrición en los niños principalmente.

- Agricultura más rentable y competitiva, creando nuevas oportunidades para la población de escasos recursos para aumentar sus ingresos.

- Agricultura más sostenible, causando menos daño al medio ambiente, al concientizar a los campesinos del trato adecuado al medio ambiente se podrá disminuir en gran porcentaje la degradación y el daño que se está ocasionando no solo al suelo sino igualmente a la atmosfera.

- Más resistente, adaptándose a condiciones de cultivo más adversas causadas por la continua degradación de la tierra y el cambio climático.

- Más equitativa, brindando nuevas oportunidades para las mujeres en zonas rurales y otros grupos marginados. 
Pueda que la "eco-eficiencia" no sea un movimiento tan fuerte como la "Revolución Verde", pero es exactamente lo que la agricultura tropical necesita para hacer frente con éxito a los intimidantes retos del siglo XXI. La combinación de variedades mejoradas de cultivos y fertilizantes químicos aportó una potente fuerza impulsora para la Revolución Verde del siglo pasado. Pero una nueva transformación de la agricultura tropical en este siglo requiere de soluciones más holísticas al problema de la baja fertilidad del suelo, soluciones que reconocen mejor las realidades económicas, sociales y ambientales de la agricultura a pequeña escala, formado de esta manera una agricultura multifuncional donde se tenga presente los impactos de la agricultura en el estado medioambiental de áreas rurales, el paisaje rural, la biodiversidad de las tierras con vocación agropecuaria y sus alrededores, la contribución de la agricultura a la viabilidad socio-económica del campo y empleo rural, la seguridad alimentaria tanto a nivel regional como nacional, el bienestar de los animales destinados a la producción, y la cultura de los agricultores y su herencia histórica.

\section{BIBLIOGRAFÍA}

1. Agudelo C. ¿cómo se hace un ensayo?, Colombia, 2005.

2. Barraza L. Perception of social and environmental problems by English and Mexican school children. Canadian Journal of Environmental Education 6: 139157. 2001.

3. Botero P. Suelos Paisaje y actividad Antrópica; Universidad Nacional de Colombia, Bogotá, 2001.

4. CIAT. Informe anual, un imperativo de eco-eficiencia para la agricultura tropical, Colombia, 2009.

5. CORPAMBU. Propuesta de ordenamiento ambiental de las áreas protectoras de Villavicencio, Villavicencio, 2008.

6. Corrales E. Sostenibilidad agropecuaria y sistemas de producción campesinos, Colombia, 2001.

7. Cuellar $\mathrm{H}$. Informe estado de los recursos naturales y del medio ambiente del municipio de Villavicencio, Villavicencio, 2009.

8. Fandiño M. C., Ferreira P. (Editoras). Colombia biodiversidad siglo XXI: Propuesta técnica para la formulación de un plan de acción ambiental en biodiversidad. 1998.

9. Instituto Geográfico Agustín Codazzí. (IGAC) Atlas de la Distribución de la Propiedad Rural en Colombia- Estadísticas - 2000-2009. p 540 Bogotá 2012. 10. Lombo R. Ecología y usos del suelo, IGAC, 1998. 
11. Ministerio de Medio Ambiente, Vivienda y Desarrollo Territorial, Resolución número 2350, Bogotá, 3 de diciembre del 2009.

12. Malagón D. Los suelos de Colombia, Instituto Geográfico Agustín Codazzi, IGAC, Cali, 2002.

13. Marcos Z. O solo nos ciclos da naturaleza. In XIII Congreso Latinoamericano de ciencia do solo. Aguas de Lindota, S.P., Brasil, 4-8 agosto de 1996. Sociedade Brasileira da Ciência do Solo. Sao Paulo, Sonopress, CD-ROM. p. 1-18. 1996.

14. Newhouse N. Implications of attitude and behavior research for environmental conservation Journal of Environmental Education. 22: 26-32. 1990.

15. Rivas L., Hoyos P., Amézquita M. Análisis económico de una estrategia para su conservación y mejoramiento, Cali, 2004.

16. Rodríguez M. Indicadores ambientales para el uso del suelo. Cuba, 2005. 